\title{
Diversidade De Leguminosae nas CaAtingas de TuCano, BaHia: IMPLICAÇÕES PARA A FITOGEOGRAFIA DO SEMI-ÁRIDO DO NORDESTE DO BRASIL ${ }^{1}$
}

\author{
Domingos Benício Oliveira Silva Cardoso ${ }^{2,3}$ \& Luciano Paganucci de Queiroz ${ }^{2}$
}

\begin{abstract}
RESUMO
(Diversidade de Leguminosae nas caatingas de Tucano, Bahia: implicações para a fitogeografia do semi-árido do Nordeste do Brasil) A família Leguminosae é a mais diversa nas caatingas em termos de número de espécies, as quais apresentam diferentes padrões de distribuição geográfica que têm permitido inferir relações florísticas entre diferentes áreas. Este trabalho apresenta o levantamento das Leguminosae nas caatingas da região de Tucano com o objetivo de avaliar a similaridade florística entre essas caatingas com a de outras áreas do semi-árido brasileiro. Para tanto, foram realizadas 11 excursões para nove áreas de caatinga, de 2004 a 2006, e realizada uma análise de similaridade entre 24 áreas usando o índice de Sørensen e agrupamento por UPGMA. Foram amostradas 74 espécies pertencentes a 41 gêneros. As análises de similaridade mostraram que as caatingas de Tucano, apesar de geograficamente próximas, não foram agrupadas entre si, associandose, por outro lado, com outras áreas de caatinga localizadas sobre substratos semelhantes. Tais resultados mostram a importância do uso de uma escala local em estudos fitogeográficos e reforçam a hipótese de que a vegetação do semi-árido é constituída por duas biotas distintas, uma relacionada a áreas sobre sedimentos arenosos e outra a áreas em solos derivados do embasamento cristalino.
\end{abstract}

Palavras-chave: Biogeografia, florestas secas, similaridade florística.

\section{Abstract}

(Leguminosae diversity in the caatinga of Tucano, Bahia: implications for phytogeography of semi-arid region of Northeastern Brazil) Leguminosae is the most diverse family in the caatinga based on the number of species. These species show distinct patterns of geographic distribution, allowing the infererence of floristic relationships between different areas. This paper presents a floristic survey of the Leguminosae in the caatinga at Tucano in order to assess floristic similarity with other areas of the Brazilian semi-arid. Eleven fieldtrips to nine caatinga sites were carried out from 2004 to 2006. Similarity analysis of 24 caatinga areas was performed using Sørensen's index and UPGMA clustering. The survey identified 74 species belonging to 41 genera. Despite closer proximity of the caatinga areas studied in Tucano, these areas were not grouped together in the cluster analysis. They were grouped with other areas of caatinga growing on similar substrate. These results highlight the importance of a local scale in phytogeographical studies and support the hypothesis that semi-arid vegetation includes two distinct biotas, one related to sedimentary sand surfaces, the other on soils derived from the crystalline rock shield.

Key words: Biogeography, dry forests, floristic similarity.

\section{INTRODUÇÃO}

As caatingas compreendem um tipo de vegetação estacional que cobre a maior parte da área com clima semi-árido da Região Nordeste do Brasil (Andrade-Lima 1981; Prado 2003), ocupando cerca de $800.000 \mathrm{~km}^{2}$ (Ab'Sáber 1974), principalmente em altitudes mais baixas, entre planaltos e serras. Na Bahia, as caatingas distribuem-se em quase toda a extensão nordeste e central do estado, praticamente circundando toda a Chapada Diamantina (Queiroz et al. 2005). Apesar das caatingas formarem um dos biomas mais ameaçados da região neotropical (Janzen 1988; Pennington et al. 2006), elas têm recebido pouca atenção em termos de conservação e estão entre os mais desvalorizados e mal conhecidos botanicamente (Giulietti et al. 2002). No entanto, essa postura tem mudado nos últimos anos, após ter sido constatado que as

Artigo recebido em 08/2006. Aceito para publicação em 03/2007.

${ }^{1}$ Esse trabalho foi realizado com auxílio do CNPq através de bolsa de iniciação científica ao primeiro autor e de produtividade em pesquisa (Pq2) ao segundo (Processos 301488/2004-5 e 117692/2004-2).

${ }^{2}$ Universidade Estadual de Feira de Santana, Depto. de Ciências Biológicas, km 03-BR 116, Campus, 44031-460, Feira de Santana, Bahia, Brazil.

${ }^{3}$ Autor para correspondência: cardosobot@gmail.com 
caatingas são constituídas por uma flora autóctone e rica em táxons endêmicos (Prado 1991; Harley 1996; Giulietti et al. 2002; Queiroz 2006a).

As caatingas podem ser caracterizadas, em geral, como florestas de porte baixo, compreendendo principalmente árvores e arbustos que geralmente apresentam espinhos e microfilia, com presença de plantas suculentas e um estrato herbáceo efêmero, presente somente durante a curta estação chuvosa. Algumas famílias, como Leguminosae, Euphorbiaceae, Bignoniaceae e Cactaceae são muito importantes por representarem a maior parte da diversidade florística. Dentre estas, Leguminosae é a mais diversa, com 293 espécies em 77 gêneros, das quais 144 espécies são endêmicas (Queiroz 2006a). Muitos táxons de Leguminosae, como os pertencentes aos gêneros Mimosa, Acacia, Caesalpinia e Senna, contribuem para a formação dos estratos arbóreos e arbustivos que compõem a paisagem característica das caatingas (Queiroz 2006a).

Em estudos fitogeográficos, as caatingas têm sido tratadas como uma única unidade vegetacional integrante das florestas sazonalmente secas do Neotrópico (Prado 2000; Prado 2003; Pennington et al. 2000; Oliveira-Filho et al. 2006), apesar de possuir fisionomia e composição florística heterogêneas, recentemente tratadas como diferentes ecorregiões (Velloso et al. 2002). No entanto, Queiroz (2006b) argumentou que, sob a denominação de caatinga, ocorrem duas biotas distintas no semi-árido do Nordeste: (1) a das superfícies sedimentares arenosas e (2) a que ocorre sobre solos derivados do embasamento cristalino. Essa hipótese foi baseada, principalmente, em estudos que compararam áreas fisionomicamente homogêneas situadas sobre esses dois tipos de substrato.

As caatingas da região de Tucano podem ser importantes para a discussão da fitogeografia da vegetação do semi-árido porque elas estão localizadas na transição entre a bacia sedimentar do Tucano-Jatobá (solos arenosos) e o setor da Depressão
Sertaneja que inclui as áreas do pediplano do sertão central da Bahia (solos derivados do embasamento cristalino). Além disso, elas têm revelado uma diversidade florística relativamente alta, o que viabiliza a realização de estudos comparativos (Cardoso \& Queiroz, comunicação pessoal). A composição florística dessas duas áreas e análises de similaridade com outras áreas de caatinga permitirão avaliar se a proximidade geográfica entre elas conduz à formação de um conjunto florístico único ou se a composição de espécies está mais diretamente relacionada ao tipo de substrato. Dessa forma, será possível testar, em escala local, a hipótese de Queiroz (2006b) de que as caatingas sobre areia apresentam uma biota distinta daquelas sobre o embasamento cristalino.

A família Leguminosae, por apresentar (1) grande número de táxons nas caatingas, (2) diferentes padrões de distribuição (Queiroz 2002) e (3) diversificação antiga em áreas secas (Lavin et al. 2004), reúne atributos que permite usar dados de distribuição de suas espécies para inferir relações entre as diferentes unidades florísticas incluídas no semi-árido (Queiroz 2006b). Assim, o principal objetivo deste trabalho é analisar, através dos dados de distribuição das Leguminosae, a similaridade florística das diferentes formas de caatinga da região de Tucano com outras áreas do semi-árido brasileiro. Para isso, procuraremos responder às seguintes questões: (i) As espécies de Leguminosae se distribuem homogeneamente nas caatingas da região de Tucano? ou (ii) existem espécies exclusivas de áreas de areia ou do embasamento cristalino? (iii) as caatingas da região de Tucano apresentam maior similaridade com outras áreas de caatinga que ocorrem sobre embasamento cristalino (a partir daqui referidas como caatinga s.s.) ou com áreas sedimentares arenosas da Região Nordeste do Brasil? (iv) a proximidade geográfica tem uma influência maior sobre a distribuição das espécies de Leguminosae do que o tipo de substrato? 


\section{Material e Métodos}

\section{Área de estudo}

O município de Tucano está localizado no nordeste do estado da Bahia, aproximadamente entre as coordenadas $10^{\circ} 55^{\prime} \mathrm{S} 39^{\circ} 04^{\prime} \mathrm{W}$ e $12^{\circ} 01^{\prime} \mathrm{S} 38^{\circ} 38^{\prime} \mathrm{W}$, na transição entre a bacia sedimentar do Tucano-Jatobá e a Depressão Sertaneja Meridional (Fig. 1). O clima da região é do tipo árido a semi-árido, com temperatura média 20,7 a $27,1^{\circ} \mathrm{C}$, precipitação média anual de 300 a $800 \mathrm{~mm}$, sem uma estação chuvosa definida, mas às vezes ocorrendo chuvas na primavera-verão (SEI 2006). As áreas de caatinga em solos derivados do embasamento cristalino distribuem-se, em sua maior parte, na parte oeste da região de Tucano, onde são encontradas também algumas serras com afloramentos graníticos que chegam a alcançar cerca de $650 \mathrm{~m}$ de altitude. Na região leste, o clima é um pouco mais úmido, com precipitação média anual de 800 a $1100 \mathrm{~mm}$ (SEI 2006). Nessa região encontram-se áreas de caatinga sobre solos arenosos, bem como formações de arenito.

Apesar das caatingas de Tucano terem sido marcadas por um histórico recente de antropização, muitas áreas bem preservadas ainda podem ser encontradas. Em levantamentos florísticos realizados anteriormente nestas áreas, observou-se uma elevada riqueza de espécies (D.B.O.S.Cardoso \& L.P. Queiroz, dados não publicados), incluindo a presença de novos táxons para a ciência, como espécies pertencentes aos gêneros Vasconcellia (Caricaceae), Arrabidaea (Bignoniaceae) e Pseudobombax (Malvaceae), além de uma espécie de Senna que será referida neste trabalho.

\section{Amostragem florística}

A lista de espécies de Leguminosae das caatingas de Tucano foi obtida a partir da realização de onze excursões entre os anos de 2004 a 2006 em quatro áreas de caatinga situadas no embasamento cristalino e cinco em áreas de superfícies sedimentares arenosas. Além disso, foram incluídas as espécies que já haviam sido coletadas anteriormente e que estão depositadas no herbário HUEFS. A maioria das espécies foi coletada em estádio reprodutivo (com flor e/ou fruto). Todo o material coletado foi depositado na coleção do herbário HUEFS.

\section{Preparação e análise dos dados}

Para o estudo de similaridade florística, foram selecionadas 24 áreas de caatingas, incluindo tanto áreas sobre solos arenosos como sobre solos derivados do embasamento cristalino (Tab. 1). Utilizou-se apenas áreas de caatinga uma vez que outros estudos mostraram que essas áreas formam uma unidade distinta das de vegetação de cerrado, campo rupestre ou mata atlântica (OliveiraFilho et al. 2006; Queiroz 2006b).

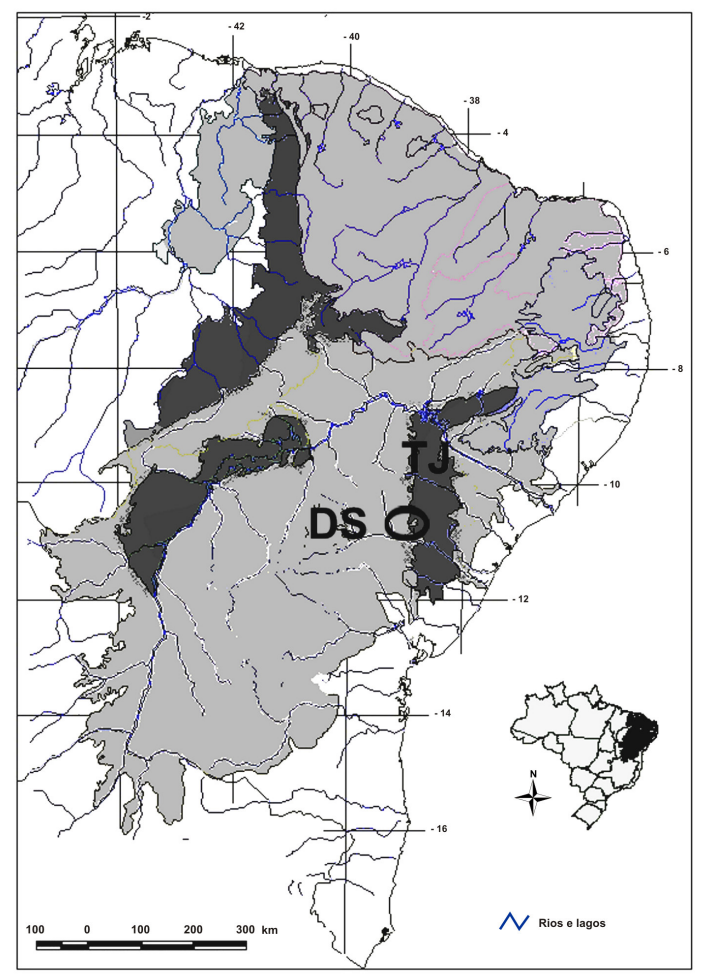

Figura 1 - Mapa da Região Nordeste do Brasil destacando a área ocupada pelas caatingas. Principais áreas de caatinga sobre sedimentos arenosos estão representadas em tom mais escuro. Um círculo indica a localização da região de Tucano mostrando que sua parte oeste encontra-se na Depressão Sertaneja Meridional (DS) enquanto a parte leste está na bacia sedimentar do Tucano-Jatobá (TJ). 
Tabela 1 - Áreas selecionadas para análise de similaridade.

\begin{tabular}{|c|c|c|c|c|}
\hline Local & Sigla & Coordenadas & Topografia e substrato & Referências \\
\hline Bom Jesus da Lapa - BA & BJLP & $13^{\circ} 24^{\prime} \mathrm{S} 43^{\circ} 21^{\prime} \mathrm{W}$ & Embasamento cristalino & $\begin{array}{l}\text { Andrade-Lima (1977); } \\
\text { L.P. Queiroz (dados não } \\
\text { publicados) }\end{array}$ \\
\hline Buíque - PE & BUIQ & $08^{\circ} 39^{\prime} \mathrm{S} 38^{\circ} 41^{\prime} \mathrm{W}$ & Bacia sedimentar arenosa & Rodal et al. (1998) \\
\hline Canudos - BA & CANU & $09^{\circ} 58^{\prime} \mathrm{S} 39^{\circ} 06^{\prime} \mathrm{W}$ & Bacia sedimentar arenosa & Queiroz (2004) \\
\hline Cariris - PB & CARI & $07^{\circ} 28^{\prime} \mathrm{S} 36^{\circ} 53^{\prime} \mathrm{W}$ & Embasamento cristalino & $\begin{array}{l}\text { Gomes (1979); Lima } \\
\text { (2004) }\end{array}$ \\
\hline Caruaru - PE & CARU & $08^{\circ} 11^{\prime} \mathrm{S} 36^{\circ} 01^{\prime} \mathrm{W}$ & Embasamento cristalino & $\begin{array}{l}\text { Alcoforado-Filho et al. } \\
\text { (2003) }\end{array}$ \\
\hline Campo Alegre de Lurdes - BA & CPAL & $09^{\circ} 30^{\prime} \mathrm{S} 43^{\circ} 05^{\prime} \mathrm{W}$ & Embasamento cristalino & Queiroz (2004) \\
\hline Casa Nova- BA & CSNV & $09^{\circ} 30^{\prime} \mathrm{S} 41^{\circ} 12^{\prime} \mathrm{W}$ & Bacia sedimentar arenosa & Queiroz (2004) \\
\hline Ibiraba - BA & IBIR & $10^{\circ} 48^{\prime} \mathrm{S} 42^{\circ} 50^{\prime} \mathrm{W}$ & Bacia sedimentar arenosa & Rocha et al. (2004) \\
\hline Ipirá - BA & IPIR & $12^{\circ} 08^{\prime} \mathrm{S} 40^{\circ} 00^{\prime} \mathrm{W}$ & Embasamento cristalino & $\begin{array}{l}\text { L.P. Queiroz (dados não } \\
\text { publicados) }\end{array}$ \\
\hline Itiúba - BA & ITIU & $10^{\circ} 21^{\prime} \mathrm{S} 39^{\circ} 36^{\prime} \mathrm{W}$ & Embasamento cristalino & Queiroz (2004) \\
\hline Jaburuna-CE & IBIA & $03^{\circ} 54^{\prime} \mathrm{S} 40^{\circ} 59^{\prime} \mathrm{W}$ & Bacia sedimentar arenosa & Araújo \& Martins 1999 \\
\hline Januária - MG & JANU & $15^{\circ} 28^{\prime} \mathrm{S} 44^{\circ} 23^{\prime} \mathrm{W}$ & Embasamento cristalino & Ratter et al. (1978) \\
\hline Maracás - BA & MARA & $13^{\circ} 23^{\prime} \mathrm{S} 43^{\circ} 21^{\prime} \mathrm{W}$ & Embasamento cristalino & $\begin{array}{l}\text { Andrade-Lima (1971); } \\
\text { L.P. Queiroz (dados não } \\
\text { publicados) }\end{array}$ \\
\hline Milagres - BA & MILA & $12^{\circ} 53^{\prime} \mathrm{S} 39^{\circ} 49^{\prime} \mathrm{W}$ & Embasamento cristalino & França et al. (1997) \\
\hline Novo Oriente - CE & NVOR & $05^{\circ} 28^{\prime} \mathrm{S} 40^{\circ} 52^{\prime} \mathrm{W}$ & Bacia sedimentar arenosa & Araújo et al. (1998) \\
\hline Ouricuri - PE & OURI & $07^{\circ} 57^{\prime} \mathrm{S} 39^{\circ} 38^{\prime} \mathrm{W}$ & Embasamento cristalino & Silva (1985) \\
\hline Remanso - BA & REMA & $09^{\circ} 33^{\prime} \mathrm{S} 42^{\circ} 05^{\prime} \mathrm{W}$ & Embasamento cristalino & Queiroz (2004) \\
\hline Raso da Catarina - BA & RSCT & $09^{\circ} 31^{\prime} \mathrm{S} 38^{\circ} 46^{\prime} \mathrm{W}$ & Bacia sedimentar arenosa & $\begin{array}{l}\text { Guedes-Bruni (1985); } \\
\text { Queiroz (2004) }\end{array}$ \\
\hline Serra da Capivara - PI & SACP & $08^{\circ} 26^{\prime} \mathrm{S} 42^{\circ} 19^{\prime} \mathrm{W}$ & Bacia sedimentar arenosa & Lemos \& Rodal (2002) \\
\hline Seridó - RN & SERI & $06^{\circ} 35^{\prime} \mathrm{S} 37^{\circ} 15^{\prime} \mathrm{W}$ & Embasamento cristalino & Camacho (2001) \\
\hline São Raimundo Nonato - PI & SRNN & $07^{\circ} 54^{\prime} \mathrm{S} 42^{\circ} 35^{\prime} \mathrm{W}$ & Embasamento cristalino & $\begin{array}{l}\text { Emperaire (1985); L.P. } \\
\text { Queiroz (dados não } \\
\text { publicados) }\end{array}$ \\
\hline Tucano - BA & TCCR & $10^{\circ} 55^{\prime} \mathrm{S} 39^{\circ} 04^{\prime} \mathrm{W}$ & Embasamento cristalino & Este trabalho \\
\hline Tucano-BA & TCAR & $12^{\circ} 01^{\prime} \mathrm{S} 38^{\circ} 38^{\prime} \mathrm{W}$ & Bacia sedimentar arenosa & Este trabalho \\
\hline Xingó - SE & XING & $09^{\circ} 35^{\prime} \mathrm{S} 37^{\circ} 35^{\prime} \mathrm{W}$ & Embasamento cristalino & Fonseca (1991) \\
\hline
\end{tabular}

A matriz de dados foi construída combinando a lista de espécies das diferentes áreas de Tucano com as listas de outras áreas de caatinga utilizadas por Queiroz (2006b). Os nomes das espécies foram atualizados usando trabalhos taxonômicos mais recentes sobre as Leguminosae. As análises de similaridade entre as áreas foram realizadas através do programa Fitopac 1.1 (Shepherd 1995). O índice de Sørensen (Mueller-Dombois \& Ellenberg 1974) foi calculado a partir da matriz binária para estimar a similaridade entre as áreas, sendo a ligação dos grupos avaliada através do método UPGMA. Análise de ordenação (PCO) foi realizada a partir da matriz de similaridade usando o mesmo programa. 


\section{Resultados}

\section{Lista de espécies}

Foram registradas 74 espécies pertencentes a 41 gêneros de Leguminosae (Tab. 2). Os gêneros com maior número de espécies são Senna (9), Mimosa (8) e Chamaecrista (6). Das espécies amostradas, 45 ocorrem nas áreas de caatinga derivadas de solos do embasamento cristalino, sendo 37 exclusivas desse tipo de ambiente. Nestas áreas, dentre as espécies mais comuns, podemos citar Anadenanthera colubrina var. cebil, Centrosema virginianum, Dioclea grandiflora, Erythrina velutina, Mimosa tenuiflora, Poeppigia procera var. conferta e Senna macranthera var. micans. Recentemente foi proposta uma nova espécie, Senna bracteosa (espécie inédita), que provavelmente é endêmica de uma serra incluída neste mesmo tipo de caatinga (Cardoso \& Queiroz, submetido).

Nas áreas de caatinga sobre solos sedimentares arenosos ocorrem 37 espécies, das quais 29 são exclusivas dessa área. Algumas espécies como Aeschynomene sensitiva, Calliandra aeschynomenoides, Copaifera cearensis var. arenicola, Galactia remansoana, Lonchocarpus araripensis, Piptadenia moniliformis e Trischidium molle ocorrem na maioria das áreas arenosas de Tucano enquanto Bauhinia corifolia (espécie inédita), Chamaecrista barbata, Hymenaea martiana e Parkia platycephala foram amostradas em apenas uma localidade cada. Mimosa brevipinna também só ocorre em uma área de areia no limite com o município de Ribeira do Pombal e era conhecida por apenas duas coletas em Oeiras (Queiroz 2002).

Apenas oito espécies foram comuns às áreas de caatinga sobre areia e sobre o embasamento cristalino, quais sejam: Acacia bahiensis, Bauhinia subclavata, Caesalpinia ferrea, Caesalpinia pyramidalis, Chaetocalyx scandens, Chamaecrista belemii var. belemii, Poecilanthe ulei e Senna acuruensis var. acuruensis.

\section{Análise de similaridade florística}

O coeficiente de similaridade de Sørensen entre as áreas de caatinga sobre areia e sobre o embasamento cristalino da região de Tucano foi de $48,4 \%$. A similaridade entre as áreas de Tucano e as outras áreas de caatinga variou entre $37,6 \%$ e $60,2 \%$. Como as análises de UPGMA e de PCO apóiam a formação dos mesmos grupos, apenas a primeira é mostrada nesse trabalho (Fig. 2). A análise de agrupamento resultou na formação de um grande grupo a $63 \%$ de similaridade, excluindo duas áreas: a de caatinga arbórea sobre solo calcáreo em Bom Jesus da Lapa e a de caatinga periodicamente inundável em Remanso. Dentro desse grande grupo, dois grupos principais, 'A' e 'B' foram formados a $67 \%$ de similaridade (Fig. 2).

$\mathrm{O}$ grupo $\mathrm{A}$ inclui todas as áreas que ocorrem em superfícies sedimentares com solos arenosos. Neste grupo, foi observado um subgrupo (A1) onde se encontra a

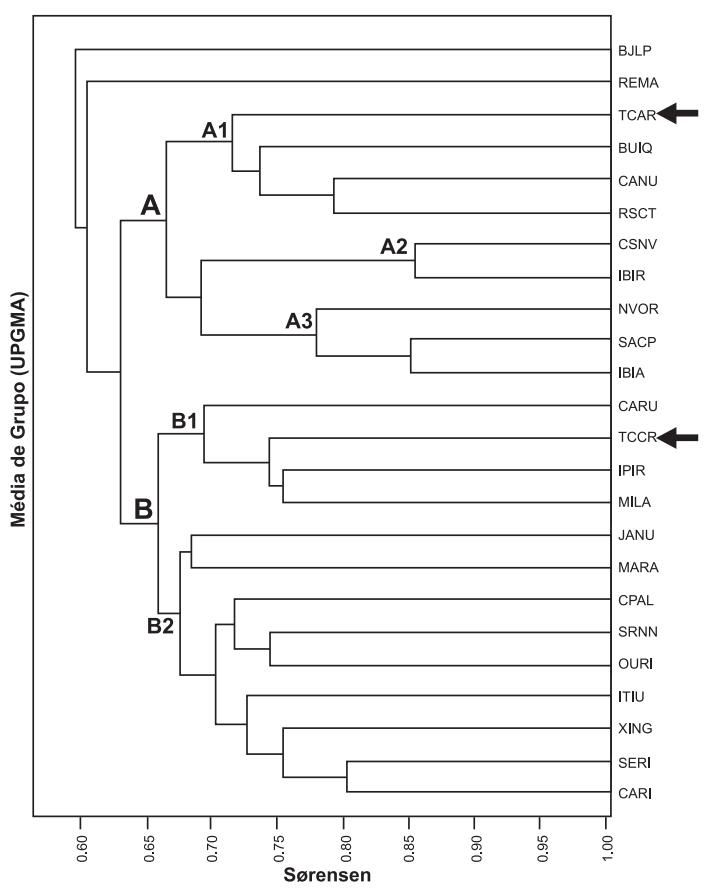

Figura 2 - Dendrograma representando a similaridade entre 24 áreas de caatinga baseado na presença de espécies de Leguminosae (ver Tabela 1 para abreviações). 
Tabela 2 - Lista de espécies de Leguminosae amostradas para as caatingas da região de Tucano (O asterisco refere-se às espécies endêmicas do bioma caatinga. Siglas para os coletores: $\mathrm{C}=\mathrm{D}$. Cardoso; LP = L.P. Queiroz; AC = A.M. Carvalho; LL = L.C.L. Lima).

\begin{tabular}{|c|c|c|c|}
\hline Espécie & Voucher & Cristalino & Areia \\
\hline * Acacia bahiensis Benth. & LP 9029; C 2, 131 & $\mathrm{x}$ & $\mathrm{x}$ \\
\hline Acacia farnesiana $($ L.) Willd. & AC 3857; LP 3117 & $\mathrm{x}$ & \\
\hline * Acacia langsdorffii Benth. & C 550 & $\mathrm{x}$ & \\
\hline Aeschynomene mollicula Kunth & C 91, 466 & $\mathrm{x}$ & \\
\hline Aeschynomene sensitiva $\mathrm{Sw}$. & C 223 & & $\mathrm{x}$ \\
\hline Anadenanthera colubrina var. cebil (Griseb.) Altschul & C 39 & $\mathrm{x}$ & \\
\hline Andira humilis Mart. & C 985 & & $\mathrm{x}$ \\
\hline * Bauhinia aculeata $\mathrm{L}$. & LP 9024; C 485 & $\mathrm{x}$ & \\
\hline Bauhinia corifolia L.P.Queiroz sp. ined. & C 219 & & $\mathrm{x}$ \\
\hline * Bauhinia subclavata Benth. & C $20,158,973$ & $\mathrm{x}$ & $\mathrm{x}$ \\
\hline Bowdichia virgilioides Kunth & LP 3711 & & $\mathrm{x}$ \\
\hline Caesalpinia ferrea Mart. ex Tul. & AC $3825 ;$ C 175 & $\mathrm{x}$ & $\mathrm{x}$ \\
\hline * Caesalpinia laxiflora Tul. & C 226 & & $\mathrm{x}$ \\
\hline * Caesalpinia pyramidalis Tul. var. pyramidalis & LP $3703 ;$ C 14,107 & $\mathrm{x}$ & $\mathrm{x}$ \\
\hline * Calliandra aeschynomenoides Benth. & C 959, 966 & & $\mathrm{x}$ \\
\hline Canavalia brasiliensis Mart. ex Benth. & C 116 & $\mathrm{x}$ & \\
\hline Centrosema arenarium Benth. & C 930 & & $\mathrm{x}$ \\
\hline Centrosema pubescens Benth. & C 896 & & $\mathrm{x}$ \\
\hline Centrosema virginianum (L.) Benth. & LP 9023; C 38 & $\mathrm{x}$ & \\
\hline Chaetocalyx blanchetiana (Benth.) Rudd & C 53 & $\mathrm{x}$ & \\
\hline Chaetocalyx scandens var. pubescens (DC.) Rudd & LP 9005; C 11 & $\mathrm{x}$ & $\mathrm{x}$ \\
\hline * Chamaecrista barbata (Nees \& Mart.) H.S.Irwin \& Barneby & C 951 & & $\mathrm{x}$ \\
\hline $\begin{array}{l}\text { * Chamaecrista belemii (H.S.Irwin \& Barneby) H.S.Irwin } \\
\text { \& Barneby var. belemii }\end{array}$ & C $141,527,898$ & $\mathrm{x}$ & $\mathrm{x}$ \\
\hline * Chamaecrista brevicalyx (Benth.) H.S.Irwin \& Barneby & C 1189 & & $\mathrm{x}$ \\
\hline Chamaecrista repens var. multijuga (Benth.) H.S.Irwin \& Barneby & C 122,889 & & $\mathrm{x}$ \\
\hline Chamaecrista serpens (L.) Greene & C 763, 909, 980 & $\mathrm{x}$ & \\
\hline * Chamaecrista swainsonii (Benth.) H.S.Irwin \& Barneby & C 206,1185 & & $\mathrm{x}$ \\
\hline Chloroleucon foliolosum (Benth.) G.P.Lewis & C 134 & $\mathrm{x}$ & \\
\hline * Copaifera cearensis var. arenicola Ducke & C 982,1188 & & $\mathrm{x}$ \\
\hline Crotalaria holosericea Nees \& Mart. & C 108 & $\mathrm{x}$ & \\
\hline Desmanthus pernambucanus (L.) Thell. & C 71 & $\mathrm{x}$ & \\
\hline * Dioclea grandiflora Mart. ex Benth. & C 46,492 & $\mathrm{x}$ & \\
\hline Erythrina velutina Willd. & C 881 & $\mathrm{x}$ & \\
\hline * Galactia remansoana Harms & C 964 & & $\mathrm{x}$ \\
\hline Geoffroea spinosa Jacq. & LP 9000 & $\mathrm{x}$ & \\
\hline * Goniorrhachis marginata Taub. & C 510 & $\mathrm{x}$ & \\
\hline Hymenaea martiana Hayne & C 922 & & $\mathrm{x}$ \\
\hline Indigofera suffruticosa P.Mill. & C 555 & $\mathrm{x}$ & \\
\hline Inga sp. & C 933 & & $\mathrm{x}$ \\
\hline * Lonchocarpus araripensis Benth. & C 188 & & $\mathrm{x}$ \\
\hline Mimosa acutistipula (Mart.) Benth. & C 79, 157 & $\mathrm{x}$ & \\
\hline Mimosa arenosa (Willd.) Poir. & C 128 & $\mathrm{x}$ & \\
\hline
\end{tabular}




\begin{tabular}{|c|c|c|c|}
\hline Espécie & Voucher & Cristalino & Areia \\
\hline * Mimosa brevipinna Benth. & C 194, 986 & & $\mathrm{x}$ \\
\hline Mimosa lewisii Barneby & LP 3704 & $\mathrm{x}$ & \\
\hline * Mimosa ophthalmocentra Mart. ex Benth. & AC 3923 & $\mathrm{x}$ & \\
\hline Mimosa quadrivalvis var. leptocarpa (DC.) Barneby & LL 182 & $\mathrm{x}$ & \\
\hline Mimosa sensitiva L. var. sensitiva & C 130; LL 181 & $\mathrm{x}$ & \\
\hline Mimosa tenuiflora (Willd.) Poir. & C 90 & $\mathrm{x}$ & \\
\hline Parkia platycephala Benth. & C 560 & & $\mathrm{x}$ \\
\hline Parkinsonia aculeata $\mathrm{L}$. & LP $3113 ;$ C 82 & $\mathrm{x}$ & \\
\hline *Peltogyne pauciflora Benth. & C 907, 277 & & $\mathrm{x}$ \\
\hline Periandra mediterranea (Vell.) Taub. & C 184 & & $\mathrm{x}$ \\
\hline Piptadenia moniliformis Benth. & LP 3705 & & $\mathrm{x}$ \\
\hline * Piptadenia stipulacea (Benth.) Ducke & LP 3115 & $\mathrm{x}$ & \\
\hline * Pithecellobium diversifolium Benth. & AC 3882; LP 4562 & & $\mathrm{x}$ \\
\hline * Poecilanthe ulei (Harms) Arroyo \& Rudd & C 478,891 & $\mathrm{x}$ & $\mathrm{x}$ \\
\hline Poeppigia procera var. conferta Benth. & C 6, 921 & $\mathrm{x}$ & \\
\hline * Senna acuruensis (Benth.) H.S.Irwin \& Barneby var. acuruensis & C 120,949 & $\mathrm{x}$ & $\mathrm{x}$ \\
\hline Senna alata (L.) Roxb. & C 979 & $\mathrm{x}$ & \\
\hline * Senna bracteosa D.Cardoso \& L.P.Queiroz sp. ined. & C 1306,874 & $\mathrm{x}$ & \\
\hline Senna macranthera var. micans (Nees) H.S.Irwin \& Barneby & C 72, 1309 & $\mathrm{x}$ & \\
\hline Senna occidentalis (L.) Link & AC 3869 & $\mathrm{x}$ & \\
\hline Senna pendula (Willd.) H.S.Irwin \& Barneby & C 1322 & $\mathrm{x}$ & \\
\hline * Senna rizzini H.S.Irwin \& Barneby & $\mathrm{C} 4,118$ & & $\mathrm{x}$ \\
\hline Senna splendida var. gloriosa H.S.Irwin \& Barneby & C 32 & & $\mathrm{x}$ \\
\hline Senna uniflora (P.Mill.) H.S.Irwin \& Barneby & LP 9019; C 61 & $\mathrm{x}$ & \\
\hline Stylosanthes scabra Vogel & C 83 & $\mathrm{x}$ & \\
\hline Swartzia apetala var. subcordata Cowan & C 180,989 & & $\mathrm{x}$ \\
\hline Tephrosia purpurea (L.) Pers. & C 965 & & $\mathrm{x}$ \\
\hline * Trischidium molle (Benth.) H.E.Ireland & C 37 & & $\mathrm{x}$ \\
\hline Vigna peduncularis (Kunth) Fawc. \& Rendle & C 89 & $\mathrm{x}$ & \\
\hline * Zornia echinocarpa (Moric.) Benth. & C 962 & & $\mathrm{x}$ \\
\hline Zornia glabra Desv. & C 94 & $\mathrm{x}$ & \\
\hline Zornia myriadena Benth. & C 534 & $\mathrm{x}$ & \\
\hline
\end{tabular}

caatinga de areia de Tucano mostrando uma maior similaridade com as áreas de Buíque, Canudos e Raso da Catarina. Dois outros subgrupos agruparam as áreas de caatinga em Casa Nova e Ibiraba (A2) e as áreas de Novo Oriente, Ibiapaba e Serra da Capivara (A3). As três últimas áreas ocorrem em solos arenosos sobre serras e são comumente denominadas de carrasco, enquanto que as áreas de Casa Nova e Ibiraba estão ao longo do Vale do Médio São Francisco, e ocorrem sobre dunas.
O grupo B compreende todas as áreas de caatinga sobre solos derivados do embasamento cristalino pré-cambriano. A área de Tucano agrupou-se com as caatingas de Caruaru, Milagres e Ipirá (B1), todas elas incluindo significativos afloramentos de granito e gnaisse. O outro subgrupo (B2) reuniu as demais áreas de caatinga associadas ao embasamento cristalino, incluindo áreas de caatinga arbórea, de caatinga arbustiva e áreas inundáveis. 


\section{Discussão}

\section{Diversidade de espécies}

O número de espécies de Leguminosae nas caatingas de Tucano, mesmo considerando cada uma das áreas separadamente, foi relativamente alto quando comparado com outros levantamentos já realizados na Região Nordeste do Brasil, tanto em áreas do embasamento cristalino (Andrade-Lima 1977, Fonseca 1991, França et al. 1997, Camacho 2001, Alcoforado-Filho et al. 2003), bem como nas caatingas sobre solos arenosos (Rodal et al. 1998; Araújo et al. 1998, 1999; Lemos \& Rodal 2002; Pereira et al. 2002; Rocha et al. 2004; Farias \& Castro 2004).

Queiroz (2006b) destacou que a importância da família Leguminosae nas caatingas está relacionada não só à riqueza de espécies mas também ao fato dela contribuir com o maior número de espécies endêmicas. Em Tucano foi verificado que das 74 espécies amostradas, 27 (36\%) são consideradas endêmicas do bioma caatinga (Tab. 1), ocorrendo nos dois tipos de substratos. Espécies de outras famílias também exemplificam a elevada taxa de táxons endêmicos da caatinga ocorrendo na região de Tucano (Cardoso \& Queiroz, comunicação pessoal), algumas ocorrendo exclusivamente sobre solos arenosos (e.g. Anemopaegma laeve DC., Bignoniaceae, Pilosocereus tuberculatus (Wederm.) Byles \& Rowles e Tacinga inamoena (K.Schum.) N.P.Taylor \& Stuppy, Cactaceae), e outras apenas em solos derivados do embasamento cristalino (e.g. Spondias tuberosa Arruda, Anacardiaceae, Aspidosperma pyrifolium Mart., Apocynaceae, Cereus jamacaru DC., Cactaceae, Capparis cynophallophora L., Capparaceae, e Ziziphus joazeiro Mart., Rhamnaceae).

A riqueza taxonômica e o número elevado de táxons endêmicos demonstram que a flora das caatingas não deve ser vista como derivada da de outros biomas, como o Chaco e a Floresta Atlântica, como defendido por Rizzini (1979). De fato, esses resultados reforçam a opinião de que as caatingas apresentam uma vegetação autóctone, como argumentado mais recentemente por vários autores (Prado 1991; Harley 1996; Giulietti et al. 2002; Queiroz 2006a), embora reconhecendo a heterogeneidade ambiental contida nesse bioma.

\section{Relações florísticas}

Estudos fitogeográficos realizados em escala continental baseados nos padrões de distribuição de espécies arbóreas têm mostrado que a caatinga faz parte da Província das Florestas Tropicais Sazonalmente Secas (SDTF) (Prado 2000; Pennington et al. 2000; Oliveira-Filho et al. 2006). Deve-se ressaltar que, nesses estudos, o bioma caatinga é considerado como uma única unidade de análise. No entanto, das 57 espécies lenhosas de caatinga usadas por Pennington et al. (2000) para a delimitação das SDTF, somente Commiphora leptophloeos (Mart.) J.G.Gillett (Burseraceae) foi encontrada nas dunas de Ibiraba (Rocha et al. 2004). Além disso, as espécies incluídas naquela província fitogeográfica raramente são encontradas em outras áreas arenosas do bioma caatinga (e.g. Araújo et al. 1998, 1999; Rodal et al. 1998; Nascimento et al. 2003; Queiroz et al. 2005). No município de Tucano, por exemplo, das 17 espécies de Leguminosae citadas por Pennington et al. (2000) somente Hymenaea martiana ocorre nas áreas arenosas, enquanto Poeppigia procera var. conferta, Geoffroea spinosa e Anadenanthera colubrina var. cebil só foram encontradas nas áreas do embasamento cristalino. Como foi verificado na Figura 2, a similaridade florística entre as diversas fisionomias de vegetação de caatinga também mostrou que as duas áreas de Tucano foram agrupadas separadamente, refletindo a presença de poucas espécies compartilhadas entre elas. Ao contrário, as áreas sobre solo arenoso agruparam-se com outras áreas 
sobre o mesmo tipo de substrato (grupo A, Fig. 2), o mesmo ocorrendo com as áreas localizadas sobre substratos derivados do embasamento cristalino (grupo B). Esses resultados podem indicar que a caatinga de Tucano é, na verdade, constituída por duas biotas distintas.

Recentemente, Queiroz (2006b), baseado no padrão de distribuição de Leguminosae e na análise de similaridade entre vários tipos de vegetação do Nordeste do Brasil, utilizando também apenas as espécies dessa família, reconheceu que as caatingas formam um grande bloco florístico mas com duas unidades fortemente distintas: (a) uma associada com o embasamento cristalino, cobrindo a maior parte da região de caatinga e mais relacionada com as SDTF e (b) a outra que ocupa as superfícies arenosas sedimentares descontínuas, apresentando uma flora bem característica e com uma proporção relativamente alta de táxons que ocorrem disjuntamente entre elas.

Em escala local, esse padrão proposto por Queiroz (2006b) foi também observado nas caatingas de Tucano. A parte oeste de Tucano está associada ao embasamento cristalino e agrupou-se com todas as áreas que ocorrem neste mesmo tipo de substrato (subgrupo B1, Fig. 2), na qual ocorrem extensos afloramentos de granito e gnaisse. Esse subgrupo é caracterizado principalmente pela presença de Dioclea grandiflora, Caesalpinia pyramidalis, Vigna peduncularis, Anadenathera colubrina var. cebil, Piptadenia stipulacea e Zornia myriadena. Por sua vez, as áreas de caatinga arenosa ocorrem na parte leste de Tucano e formaram um grupo bem consistente com as áreas de Buíque, Canudos e Raso da Catarina (A1, Fig. 2). Este grupo compreende as áreas da bacia sedimentar do Tucano-Jatobá, a qual se estende por quase toda o nordeste do estado da Bahia até o centro sul de Pernambuco. Das seis espécies que foram consideradas por Queiroz (2006b) como sendo endêmicas dessa formação, três delas ocorrem em Tucano: Calliandra aeschynomenoides, Chamaecrista swainsonii e Zornia echinocarpa. Além disso, algumas espécies que, em Tucano, só ocorrem na caatinga arenosa, como Piptadenia moniliformis, Trischidium molle, Chamaecrista repens var. multijuga, Lonchocarpus araripensis e Copaifera cearensis var. arenicola, apresentam distribuição disjunta com outras áreas de caatinga da Região Nordeste que também ocorrem sobre sedimentos arenosos (Queiroz 2006b).

A definição de uma escala local em estudos fitogeográficos é importante porque o grau de similaridade entre áreas pode estar sujeito a um gradiente ambiental e, nesse caso, uma escala mais detalhada permite reconhecer conjuntos florísticos distintos (Nekola \& White 1999). No caso das áreas de Tucano, a separação dos dados de acordo com o tipo de ambiente permitiu testar se a proximidade geográfica teve uma influência maior sobre a distribuição das espécies de plantas do que o tipo de substrato. $\mathrm{O}$ fato das áreas amostradas de Tucano não formarem um grupo entre si refuta a hipótese de que a proximidade geográfica é a causa principal da distribuição das espécies nessa região. No entanto, o agrupamento das duas áreas de Tucano com outras áreas situadas sobre o mesmo tipo de substrato reforça a hipótese de que esse é o principal fator causal da distribuição das espécies de plantas na caatinga.

Por outro lado, a falta de uma escala apropriada para análises fitogeográficas a nível local pode trazer problemas para a discriminação de conjuntos florísticos distintos que ocorrem em áreas geograficamente próximas. Por exemplo, no caso da área de Remanso, verifica-se que a vegetação é heterogênea, ocorrendo em áreas sobre solo arenoso, em planícies aluviais e sobre afloramentos do embasamento cristalino (L.P.Queiroz, dados não publicados). $\mathrm{Na}$ análise de similaridade realizada no presente 
estudo, os dados de Remanso não foram coletados de forma separada por ambiente, o que resultou em uma grande dissimilaridade dessa área em relação às demais áreas de caatinga analisadas. Deste modo, poderíamos supor que uma análise mais refinada, separando os ambientes de Remanso, poderia demonstrar a natureza composta de sua biota, como foi verificado no presente trabalho para a região de Tucano.

O padrão de diferenciação local da vegetação das caatingas de Tucano reforça, portanto, as hipóteses biogeográficas apresentadas por Queiroz (2006b) de que as áreas de caatinga arenosa representam uma unidade fitogeográfica distinta da caatinga s.s. ou de outra floresta seca neotropical que ocorre sobre o embasamento cristalino. Além do padrão de distribuição das plantas, essa hipótese é reforçada por dados fenológicos de deciduidade foliar e de floração. Por exemplo, Rocha et al. (2004) verificaram que nas regiões das dunas do Rio São Francisco, em Ibiraba, Bahia, pelo menos $50 \%$ das plantas lenhosas retêm as folhas mesmo nos meses mais secos e há flores de diferentes espécies em antese ao longo do ano. Esse padrão fenológico é bem diferente do observado nas áreas de caatinga sobre o embasamento cristalino, em que a proporção de espécies que mantém as folhas na estação seca é de $26 \%$ e a floração é concentrada no início da estação chuvosa (Machado et al. 1997). Apesar de não se ter realizado um estudo fenológico quantitativo na região de Tucano, verificouse, ao longo desse trabalho, que a maioria das espécies de caatinga sobre substrato arenoso mantinham suas folhas na estação seca, situação distinta daquelas que crescem sobre o embasamento cristalino, pois a quase totalidade dessas perde as folhas no mesmo período.

Em trabalho recente sobre a fitogeografia das florestas sazonalmente secas do leste da América do Sul, Oliveira-
Filho et al. (2006) também verificaram, como, no presente estudo, que áreas de caatinga s.s. foram agrupadas em um bloco distinto das áreas comumente denominadas de carrasco, embora eles considerem que a caatinga seja considerada como um único núcleo florístico das SDTF. Esses autores explicaram a dicotomia das áreas de caatinga s.s. e de carrasco pela sua associação, respectivamente, a solos derivados do embasamento cristalino ou a depósitos arenosos (Araújo et al. 1998; Rodal \& Sampaio 2002; Oliveira-Filho et al. 2006). Outra explicação para a baixa similaridade florística entre essas áreas de caatinga s.s. e carrasco observada em escala continental (Oliveira Filho et al. 2006), regional (Queiroz 2006b) e na região de Tucano (presente trabalho) é a possibilidade delas representarem unidades fitogeográficas distintas formadas por diferentes processos históricos (Queiroz 2006b) e, portanto, não deveriam ser tratadas genericamente com o termo de caatinga ou como uma única unidade vegetacional.

\section{Agradecimentos}

Os autores agradecem ao Sr. Olavo, Antônio da Bila, Timóteo, Marcos Guerreiro, George, Alexandre, Ademário e Quézia pela ajuda no trabalho de campo, e ao Sr. João Cacimba e Jeová por terem oferecido alojamento durante as excursões para a Serra das Candeias.

\section{REFERÊNCIAS BibLIOGRÁFICAS}

Ab'Saber, A. N. 1974. O domínio morfoclimático semi-árido das caatingas brasileiras. Geomorfologia 43: 1-39.

Alcoforado-Filho, F. G.; Sampaio, E. V. S. B. \& Rodal, M. J. N. 2003. Florística e fitossociologia de um remanescente de vegetação caducifólia espinhosa arbórea em Caruaru, Pernambuco. Acta Botanica Brasilica 17(2): 287-303. 
Andrade-Lima, D. 1971. Vegetação da área Jaguaquara-Maracás, Bahia. Ciência e Cultura 23(3): 317-319. 1977. Flora de áreas erodidas de calcário Bambuí, em Bom Jesus da Lapa, Bahia. Revista Brasileira de Biologia 37: 179-194. 1981. The caatingas dominium. Revista Brasileira de Botânica 4: 149-153.

Araújo, F. S.; Sampaio, E. V. S. B.; Figueiredo, M. A.; Rodal, M. J. N. \& Fernandes, A. F. 1998. Composição florística da vegetação de carrasco, Novo Oriente, CE. Revista Brasileira de Botânica 21(2): 105-116.

\& Martins, F. R. 1999. Fisionomia e organização da vegetação do carrasco no Planalto da Ibiapaba, estado do Ceará. Acta Botanica Brasilica 13(1): 1-14.

Camacho, R. G. V. 2001. Estudo fitofisiográfico da caatinga do Seridó - Estação Ecológica do Seridó, RN. Tese de Doutorado. Universidade de São Paulo, São Paulo, 130p.

Cardoso, D. B. O. S. \& Queiroz, L. P. A new species of Senna P. Miller (Leguminosae, Caesalpinioideae) from Northeastern Brazil. Novon. (Submetido).

Emperaire, L. 1985. Végétation de l'État du Piauí (Brésil). Compte Rendu des Seances de la Societé de Biogeographie 60(4): 151-163.

Farias, R. R. S. \& Castro, A. A. J. F. 2004. Fitossociologia de trechos da vegetação do Complexo de Campo Maior, Campo Maior, PI, Brasil. Acta Botanica Brasilica 18(4): 949-963.

Fonseca, M. R. 1991. Análise da vegetação arbustiva-arbórea da caatinga hiperxerófila do noroeste do estado de Sergipe. Tese de Doutorado. Universidade Estadual de Campinas, Campinas, 187p.

França, F.; Melo, E. de \& Santos, C. C. 1997. Flora de inselbergs da região de Milagres, Bahia, Brasil: I. caracterização da vegetação de lista de espécies de dois Inselbergs. Sitientibus 17: 163-184.

Giulietti, A. M.; Harley, R. M.; Queiroz, L. P.; Barbosa, M. R. V.; Bocage-Neta, A. L. \&
Figueiredo, M. A. 2002. Espécies endêmicas da caatinga. In: Sampaio, E. V.S. B.; Giulietti, A. M.; Virgínio, J. \& Gamarra-Rojas, C. F. L. (eds.). Vegetação e flora da caatinga. APNE/CNIP, Recife. Pp. 103-118.

Gomes, M. A. F. 1979. Padrões de caatinga nos Cariris Velhos, Paraíba. Dissertação de Mestrado. Universidade Federal Rural de Pernambuco, Recife, 88p.

Guedes-Bruni, R. R. 1985. Lista preliminar das angiospermas ocorrentes no Raso da Catarina e arredores, Bahia. Rodriguesia 62: 5-8.

Harley, R. M. 1996. Exemples of endemism and phytogeographical elements in the caatinga flora. Anais da IV Reunão Especial da SBPC, Feira de Santana. Pp. 219-227.

Janzen, D. H. 1988. Tropical dry forests. The most endangered major tropical ecosystem. In: Wilson, E. O. (ed.). Biodiversity. National Academy Press, Washington. Pp. 130-137.

Lavin, M.; Schrire, B. P.; Lewis, G. P.; Pennington, R. T.; Delgado-Salinas, A.; Thulin, M.; Hughes, C. E.; Matos, A. B. \& Wojciechowski, M.F. 2004. Metacommunity process rather than continental tectonic history better explains geographically structured phylogenies in legumes. Philosophical Transactions of the Royal Society B: Biological Sciences 359(1450): 1509-1522.

Lemos, J. R. \& Rodal, M. J. N. 2002. Fitossociologia do componente lenhoso de um trecho de vegetação de caatinga no Parque Nacional da Serra da Capivara, Piauí, Brasil. Acta Botanica Brasilica 16(1): 23-42.

Lima, I. B. 2004. Levantamento florístico da Reserva Particular do Patrimônio Natural Fazenda Almas, São José dos Cordeiros - PB. Monografia de Bacharelado. Universidade Federal da Paraíba, João Pessoa, 31p.

Machado, I. C.; Barros, L. M. \& Sampaio, E. V. S. B. 1997. Phenology of the caatinga species at Serra Talhada, PE, Northeastern Brazil. Biotropica 29: 57-68. 
Mueller-Dombois, D. \& Ellenberg, H. 1974. Aims and methods of vegetation ecology. John Wiley \& Sons, New York, 547p.

Nascimento, C. E. S., Rodal, M. J. N. \& Cavalcanti, A. C. 2003. Phytosociology of the remaining xerophytic woodland associated to an enviromental gradient at the banks of the São Francisco river Petrolina, Pernambuco, Brasil. Revista Brasileira de Botânica 26(3): 271-287.

Nekola, J. C. \& White, P. S. 1999. The distance decay of similarity in biogeography and ecology. Journal of Biogeography 26: 867-878.

Oliveira-Filho, A. T.; Jarenkow, J. A. \& Rodal, M. J. N. 2006. Floristic relationships of seasonally dry forests of eastern South America based on tree species distribution patterns. In: Pennington, R. T.; Lewis, G. P. \& Ratter, J. A. (eds.). Neotropical savannas and dry forests: Plant diversity, biogeography, and conservation. Taylor \& Francis CRC Press, Oxford. Pp. 59-192.

Pennington, R. T.; Prado, D. E. \& Pendry, C. A. 2000. Neotropical seasonally dry forests and Quaternary vegetation changes. Journal of Biogeography 27: 261-273.

; Lewis, G. P. \& Ratter, J. A. 2006. An overview of the plant diversity, biogeography and conservation of neotropical savannas and seasonally dry forests. In: Pennington, R. T.; Lewis, G. P. \& Ratter, J. A. (eds.). Neotropical savannas and dry forests: Plant diversity, biogeography, and conservation. Taylor \& Francis CRC Press, Oxford. Pp. 1-29.

Pereira, I. M.; Andrade, L. A.; Barbosa, M. R. V. \& Sampaio, E. V. S. B. 2002. Composição florística e análise fitossociológica do componente arbustivo arbóreo de um remanescente florestal no agreste paraibano. Acta Botanica Brasilica 16(3): 357-369.

Prado, D. E. 1991. A critical evaluation of the floristic links between chaco and caatinga vegetation in South America. Tese de
Doutorado. University Saint Andrews, Saint Andrews, 173p.

2000. Seasonally dry forests of tropical South America: from forgotten ecosystems to a new phytogeographic unit. Edinburgh Journal of Botany 57(3): 437-461.

2003. As caatingas do Brasil. In: Leal, I. R.; Tabarelli, M. \& Silva, J. M. C. (eds.). Ecologia e conservação da caatinga. Ed. Universidade Federal de Pernambuco, Recife. Pp. 3-73.

Queiroz, L. P. 2002. Distribuição de espécies de Leguminosae na caatinga. In: Sampaio, E. V. S. B.; Giulietti, A. M.; Virgínio, J. \& Gamarra-Rojas, C. F. L. (eds.). Vegetação e flora da caatinga. APNE/ CNIP, Recife. Pp. 141-153. 2004. Biodiversidade da família Leguminosae na caatinga da Bahia: florística, biogeografia e disseminação. Relatório Técnico, Universidade Estadual de Feira de Santana, Feira de Santana, 321p.

.2006a. Flowering plants of the Brazilian semi-arid. In: Queiroz, L. P.; Rapini, A. \& Giulietti, A. M. (eds.). Towards greater knowledge of the Brazilian semi-arid biodiversity. Ministério da Ciência e Tecnologia, Brasília. Pp. 49-53. 2006b. The Brazilian caatinga: phytogeographical patterns inferred from distribution data of the Leguminosae. In: Pennington, R. T.; Lewis, G. P. \& Ratter, J. A. (eds.). Neotropical savannas and dry forests: Plant diversity, biogeography, and conservation. Taylor \& Francis CRC Press, Oxford. Pp. 113-149.

; França, F.; Giulietti, A. M.; Melo, E.; Gonçalves, C. N.; Funch, L. S.; Harley, R. M.; Funch, R. R. \& Silva, T. S. 2005. Caatinga. In: Juncá, F. A.; Funch, L. S. \& Rocha, W. (eds.). Biodiversidade e conservação da Chapada Diamantina. Ministério do Meio Ambiente, Brasília. Pp. 95-120.

Ratter, J. A.; Askew, G. P.; Montgomery, R. F. \& Gifford, D. R. 1978. Observations on 
forests of some mesotrophic soils in central Brazil. Revista Brasileira de Botânica 1: 47-58.

Rizzini, C. T. 1979. Tratado de fitogeografia do Brasil: aspectos sociológicos e florísticos. Vol. 2. HUCITEC, São Paulo, 374p.

Rocha, P. L. B.; Queiroz, L. P. \& Pirani, J. R. 2004. Plant species and habitat structure in a sand dune field in the Brazilian Caatinga: a homogenous habitat harbouring an endemic biota. Revista Brasileira de Botânica 27(4): 739-755.

Rodal, M. J. N.; Andrade, K. V. A.; Sales, M. F. \& Gomes, A. P. S. 1998. Fitossociologia do componente lenhoso de um refúgio vegetacional no município de Buíque, Pernambuco. Revista Brasileira de Botânica 58(3): 517-526.

\& Sampaio, E. V. S. B. 2002. A vegetação do bioma caatinga. In: Sampaio, E. V. S. B.; Giulietti, A. M.; Virgínio, J. \& Gamarra-Rojas, C. F. L. (eds.). Vegetação e flora da caatinga. APNE/ CNIP, Recife. Pp. 11-24.
SEI (Superintendência de Estudos Econômicos e Sociais do Estado da Bahia). 2006. Banco de dados geo-ambientais. [on line] Disponível na internet via http:// www.sei.ba.gov.br. Acesso em 20 de Abril de 2006.

Silva, G. C. 1985. Flora e vegetação das depressões inundáveis da região de Ouricuri - PE. Dissertação de Mestrado. Universidade Federal Rural de Pernambuco, Recife, 180p.

Shepherd, G. J. 1995. Fitopac 1 (programa e manual). Departamento de Botânica, Universidade Estadual de Campinas, Campinas, 93p.

Velloso, A. L.; Sampaio, E. V. S. B.; Giulietti, A. M.; Barbosa, M. R. V.; Castro, A. A. J. F.; Queiroz, L. P.; Fernandes, A.; Oren, D. C.; Cestaro, L. A.; Carvalho, A. J. E.; Pareyn, F. G. C.; Silva, F. B. R.; Miranda, E. E.; Keel, S. \& Gondim, R. S. 2002. Ecorregiões: propostas para o bioma caatinga. APNE, The Nature Conservancy do Brasil, Recife, 75p. 